\title{
Review
}

\section{Treatment burden associated with the intake of thickened fluids}

The implementation of thickened fluids in patients with dysphagia is widely considered an effective strategy for safe and physiologically improved swallow. However, there is limited evidence to suggest that this intervention reduces the risk of dysphagia-related complications including aspiration pneumonia. In addition, there is growing evidence that this approach is associated with adverse clinical effects including dehydration, malnutrition and reduced health-related quality of life. This review summarises the rationale for thickened fluids, the evidence base (or lack thereof) underpinning their use, and current guideline recommendations.

\section{Educational aims}

- To review the evidence base for thickened fluids in the management of dysphagia.

- To examine the evidence that thickened fluids reduce aspiration pneumonia.

- To provide an overview of the advantages and disadvantages of thickened fluids in the management of dysphagia.

\section{Clinical scenario}

An 86-year-old male was admitted to hospital with an infective exacerbation of his idiopathic pulmonary fibrosis and delirium. He had a past medical history significant for Guillain-Barré syndrome and gastrooesophageal reflux disease. Previously he had been assessed by a speech pathologist in the community, and prior to hospitalisation his diet consisted of a regular diet and fluids. On hospital admission he was assessed by the multidisciplinary allied health team and found to have moderate oropharyngeal dysphagia, characterised by reduced oropharyngeal coordination, reduced bolus control and variable timing of pharyngeal swallow. There were also documented clinically overt signs of aspiration
@ERSpublications

Despite being a commonly prescribed treatment, there is limited evidence to support the benefits of thickened fluids in the treatment of dysphagia. This disconnect highlights the importance of a considered approach when prescribing this intervention. https://bit.ly/3a2BDwD
Cite as: Steele SJ, Ennis SL, Dobler CC. Treatment burden associated with the intake of thickened fluids. Breathe 2021; 17: 210003 
with thin fluids. Subsequently, he was prescribed moderately thickened fluids and a purée diet. On day three of his hospitalisation, the medical team identified signs and symptoms of dehydration and the patient was commenced on small quantities of thin fluids (water only) in between meals. 4 days later the patient discharged against medical advice and subsequently did not undergo a planned instrumental swallow assessment, which was scheduled for the following day. While he was advised to remain on thickened fluids, his intense dislike of this therapy resulted in him self-upgrading to thin fluids and accepting the potential aspiration risk. The patient was followed up via telehealth by the speech pathology and medical teams. He reported tolerating a normal diet including thin fluids.

\section{Background}

Swallowing is a complex series of neuromuscular events. Impairment in any component may lead to a disorder or symptom characterised by difficulty in swallowing, termed dysphagia. Oropharyngeal dysphagia is a common condition, particularly amongst older people and those with underlying neurodegenerative conditions [1] and nonneurological (head and neck cancer) diseases. Without treatment, dysphagia is known to be associated with respiratory complications including aspiration pneumonia, malnutrition, hospital admission and increased mortality [2, 3].

There are multiple interventions that have been utilised in the management of dysphagia, which extend from texture modified foods and thickened fluids, swallowing therapy, drug therapy, neuromuscular stimulation, and botulinum toxin therapy. Screening models have also been implemented into routine clinical care, with tools such as the Acute Screening for Swallow in Stroke screening tool (ASSIST) being routinely implemented within $24 \mathrm{~h}$ post-stroke admission to identify and stratify patients according to their risk of dysphagic symptoms.

The use of modified diets to prevent aspiration and its consequences in those with dysphagia has increased dramatically in recent decades [1, 4]. Thickened fluids have become the cornerstone in treating adults with dysphagia. Thickening agents are used to modify fluid properties, with the rationale being that thick fluids have a higher viscosity and can compensate for a swallowing deficit by slowing down the flow of fluid from the mouth to the oropharynx, allowing time for glottis closure which could potentially reduce the risk of aspiration [1].

The purpose of this article is to review the advantages and disadvantages of thickened fluids in adults with dysphagia, focusing on their effectiveness to reduce aspiration pneumonia and the treatment burden associated with their use.

\section{Physiological rationale}

Thickened liquids are often recommended based on aspiration detected during clinical swallow evaluation. Physiologically, thickened fluids improve swallowing by increasing oral transit time, normalising swallowing patterns during respiration and enhancing sensory input [3]. Increasing oral transit time and the flow of fluid from the mouth to the pharynx may allow more time for airway closure.

Thickened fluids reduce aspiration detected by imaging. Trials of videofluoroscopy have demonstrated improved functional swallow and decreased aspiration with the use of thickened fluids [5-8].

However, improved swallowing physiology with thickened fluids does not necessarily translate into reduced respiratory complications. The relationship between oropharyngeal dysphagia and pneumonia is complex. Aspiration pneumonia is not an inevitable consequence of aspiration, but rather dependant on a range of interacting factors at the level of the individual and connected to the aspiration event (including the amount and type of aspirate) [9].

\section{Pneumonia}

Aspiration pneumonia refers to a lower respiratory tract infection caused by inhalation of pathogenic bacteria in oropharyngeal secretions [10]. It is an important clinical entity as it is associated with increased mortality compared with other forms of pneumonia [10].

The clinical effects of thickened fluids in adults with dysphagia on the development of pneumonia have been examined in multiple systematic reviews [11-15]. These reviews have highlighted the limited number of randomised controlled trials, small sample size and the variable quality of studies. The characteristics of patients included in the trials have typically been adults over the age of 50 years with underlying neurological conditions such as Parkinson's disease or dementia who had previously aspirated liquids; or participants after a stroke or acute brain injury. Included studies have been completed in a variety of settings ranging from inpatients in acute and subacute facilities to outpatient settings. The thickened fluid "intervention" used as a strategy to facilitate safe and efficient swallow has predominately consisted of nectar thickened fluids or honey thickened fluids. Comparator groups have typically included unlimited thin fluids.

Many of the trials of thickened fluids have methodological flaws. Participant compliance with the assigned interventions was rarely monitored and many trials demonstrated a high risk of bias, especially selection and blinding 
bias [14]. In addition, most trials compared thickened fluids to another intervention such as "chin down" posture or allowing intake of thin water between meals.

Overall, these systematic reviews have demonstrated that there is no definitive evidence to suggest that thickened fluids benefit adults with dysphagia by preventing pneumonia. A systematic review published in 2018 [11] included two multicentre randomised controlled trials in patients with dementia and Parkinson's disease [7, 16]. This found no significant decrease in risk of pneumonia with nectar thickened fluid compared with thin fluids and chin down posture $(\mathrm{RR}=0.81 ; 95 \% \mathrm{Cl}$ $0.40-1.65)$ [11]. There was also no statistically significant difference between honey thickened fluid and thin fluids and chin down posture regarding the risk of pneumonia ( $\mathrm{RR}=1.58 ; 95 \% \mathrm{Cl} 0.89-2.80)$ [11]. Based on the evidence assessed using the GRADE framework (Grading of Recommendations Assessment, Development and Evaluation system) and the assessment of the risk-benefit ratio, the authors of the systematic review made a "weak recommendation against the use of textured modified liquids" [11].

Another systematic review and meta-analysis included all study types that compared thickened fluids to either thin liquids with a safety protocol or thin water protocols [14]. Six randomised controlled studies that examined the incidence of pneumonia in thin water protocols versus thickened liquids, with a cumulative total of 135 patients, were included in a meta-analysis. This demonstrated no significant difference for pneumonia risk $(\mathrm{OR}=0.82 ; 95 \% \mathrm{Cl}$ 0.05-13.52).

The risk of developing aspiration pneumonia is possibly less through the aspiration of thin pure water than the aspiration of thickened fluids. In response to difficulties getting people to adhere to thickened fluid treatment plans and the recognition that the aspiration of pure water is relatively benign with a low risk of developing aspiration pneumonia, the Frazier free water protocol was developed [17]. Thin pure water contains less pathogenic bacteria than other fluids, e.g. fruit juices [18]. In addition, aquaporins facilitate removal of water from the airspaces after aspiration while drinking [19], thus reducing the risk of aspiration pneumonia if aspiration occurs. The free water protocol allows patients deemed to be at risk of aspiration, who would otherwise be "nil by mouth" or on thickened fluids only, to drink unlimited amounts of thin pure water. Thin pure water should only be consumed at least 30 min after a meal and only after thorough oral care. Thickened fluids are still recommended together with any meals or snacks. The protocol has the potential to increase overall fluid intake and therefore reduce the risk of dehydration and improve patients' quality of life. A systematic review found that the risk of lung complications was not increased in patients on the free water protocol compared with patients with no access to thin fluids
(OR=1.51; 95\% Cl 0.2-100.03; six studies, 206 patients), but the large confidence interval of the result indicates low certainty in the evidence [13]. The fluid intake was higher in patients with the water protocol compared with patients on thickened fluids, but the difference was not statistically significant (mean difference $179.4 \mathrm{~mL}, 95 \% \mathrm{Cl}$ -70.5 to $429.2 \mathrm{~mL}$; five studies, 148 patients). From this study, the authors concluded that in carefully selected patients with dysphagia, there is low-quality evidence indicating a free water protocol could be offered.

\section{Health-related quality of life}

Health-related quality of life (HRQoL) changes with dysphagia are significant [20]. For this reason, interventions to combat dysphagia should pay careful consideration to improving HRQoL. Clinicians should be aware of the potential negative impact thickened fluids have on HRQoL and take this into consideration when weighing up the risks and benefits of this treatment.

One systematic review identified eight studies (including two very small randomised controlled trials with 15 and 18 participants, respectively) that examined the effects of altering food (typically cutting, mashing or puréeing) or fluids (typically thickening) on quality of life [21]. The included trials showed that less modified textures (i.e. normal or nearly unmodified food/drinks) were associated with considerably better HRQoL than more modified textures.

Similar to our case scenario at the beginning of this review, it is anecdotally very common that patients choose not to comply with thickened fluid prescription [1]. Patient dissatisfaction is a contributing factor to non-compliance [22]. A qualitative descriptive study that explored experiences of individuals prescribed thickened liquids after stroke showed that patients dislike thickened fluids, suggesting this may impact adherence, hydration and quality of life [23]. One study examined the effect of thickened fluids on HRQoL via a time trade-off approach in a randomised controlled trial of different consistencies of thickened fluids [24]. Participants were willing to sacrifice on average 5 years of a 10-year lifespan in order to avoid thickened fluids [24]. Product palatability (including dissatisfaction with texture and taste), sensation of increased satiety and increased thirst with increasingly viscous fluids all appear to be contributing factors to poor tolerance of thickened fluids [23-25]. Alternative strategies also seem to be better tolerated, with one large, randomised trial that evaluated three different interventions for the treatment of aspiration demonstrating that patients preferred postural adaptation (changing body position, for example sitting up or chin down) over thickened fluids [7]. 


\section{Self-evaluation questions}

1. The free water protocol allows the patient to:
a) Drink as much thickened water as they desire.
b) Consume unthickened water in between meals.
c) Consume unthickened water at the time of their puréed meal.

2. In a patient presenting with aspiration pneumonia, thickened fluids should be:

a) Prescribed universally in all patients.

b) Never prescribed, due to the documented negative association with HRQoL.

c) Discussed with the patient and their family and a shared decisionmaking approach utilised which considers the positive and negative effects of this intervention.

3. Regarding aspiration, which of the following statements is false?

a) Thickened fluids reduce aspiration detected by videofluoroscopy imaging.

b) The relationship between dysphagia-related aspiration and pneumonia is linear, with pneumonia an inevitable consequence of aspiration.

c) The risk of developing aspiration pneumonia has been proposed to be less through the aspiration of thin pure water.

\section{Nutritional requirements}

Hospitalised patients with dysphagia are at greater risk of malnutrition and dehydration than those without dysphagia [26, 27]. A study of 25 hospital inpatients on thickened fluids found that none achieved their daily fluid requirements without additional support [28]. An aversion for thickened fluids also has implications for fluid status, with patients who have a strong dislike of thickened fluids decreasing their intake, resulting in dehydration and weight loss. The failure of thickened fluids to reduce the physiological sensation of thirst is also associated with reduced oral intake [3, 27]. Research has demonstrated that dietary and fluid intake of older adults in care homes requiring a texture modified diet are significantly less, compared with those who consume a regular diet and fluids, compromising their ability to meet their dietary and nutritional needs [29]. This finding has also been replicated in patients with dysphasia following stroke, with those who received thickened fluids not meeting their fluid requirements [30]. Thickened fluids also typically come in small units (e.g. $185 \mathrm{~mL}$ ), which may further contribute to this issue. Dehydration and malnutrition can contribute to a number of negative health conditions, including the development of infections, poor wound healing and pressure sores. Furthermore, the findings of a recent Australian study indicate that health facilities do not routinely or objectively monitor fluid intake and hydration in patients with dysphagia who are prescribed thickened fluids, despite this population being at risk of poor fluid intake and dehydration [27].

\section{Effect on bioavailability of water and medication}

Administration of medication with thickened liquid requires consideration. Bioavailability of medication is impaired with viscous substances. This results in delayed dissolution and disintegration, with the ionic charge on thickening agents negatively affecting drug release [25].

\section{Guidelines}

Clinical practice guidelines ideally are systematically developed statements that assist clinicians to provide appropriate evidence-based care. A systematic review, published in 2020, evaluated the evidence base of recommendations for the intervention of thickened fluids for patients with dysphagia after a stroke [31]. The included clinical practice guidelines were published between 2010 and 2018, and included guidelines from European, Australasian, African and North American countries. The review found that despite the limited evidence base for thickened fluids there was a consensus across all guidelines in the support of thickened fluids.

\section{Shared decision making}

Given the uncertainty of the evidence that thickened fluids reduce the risk of aspiration pneumonia, possible adverse events like dehydration, and the very high treatment burden associated with this treatment, the best course of action for an individual patient should be discussed in a shared decisionmaking approach between patient and clinician [32]. Unfortunately, patients are often not involved in the decision-making process. It is important to openly discuss the pros and cons of thickened fluids as well as a free water protocol, which will allow patients to make an informed choice about the course of action that best aligns with their preferences and values. Given the unpopularity of thickened fluids among patients with swallowing problems, many would probably not favour this treatment modality given a choice.

\section{Summary}

Despite being a commonly prescribed treatment, there is no conclusive evidence to support the clinical benefits of thickened fluids in the treatment of dysphagia. This disconnect highlights the importance of a more considered approach as to 
Table 1 Advantages and disadvantages of thickened fluids

\begin{tabular}{ll}
\hline Advantages & Disadvantages \\
\hline Improved physiological and functional swallow & No definitive evidence of reduced risk of pneumonia \\
Reduced aspiration detected by imaging & Impaired bioavailability of medications \\
& Adverse effect on HRQoL \\
& Risk of malnutrition and dehydration \\
& Reduced palatability
\end{tabular}

when modified diets should be recommended to patients. Thickened fluids are a poorly tolerated intervention and possible benefits, risks, and the impact on quality of life should be discussed to enable use of shared decision making amongst the patient, family and clinician (see table 1 for a summary of the advantages and disadvantages of thickened fluids). There is a need for high-quality evidence and review of the current guidelines regarding this intervention.

\section{Key points}

- There is no conclusive evidence to support thickened fluids to prevent dysphagia-related clinical complications, including aspiration pneumonia.

- $\quad$ Thickened fluids reduce HRQoL in patients with dysphagia.

- Clinical practice guidelines frequently support the use of thickened fluids, despite the lack of evidence underpinning this intervention.

\section{Affiliations}

\section{Simon J. Steele ${ }^{1}$, Samantha L. Ennis ${ }^{2}$, Claudia C. Dobler ${ }^{2,3}$}

${ }^{1}$ Dept of General and Acute Care Medicine, Alfred Hospital, Melbourne, Australia. ${ }^{2}$ Dept of Respiratory and Sleep Medicine, Liverpool Hospital, Sydney, Australia. ${ }^{3}$ The George Institute for Global Health, University of New South Wales, Sydney, Australia.

\section{Conflict of interest}

S.J. Steele has nothing to disclose. S.L. Ennis has nothing to disclose. C.C. Dobler has nothing to disclose.

\section{References}

1. O'Keeffe ST. Use of modified diets to prevent aspiration in oropharyngeal dysphagia: is current practice justified? BMC Geriatr 2018; 18: 167.

2. Carrión S, Cabré M, Monteis R, et al. Oropharyngeal dysphagia is a prevalent risk factor for malnutrition in a cohort of older patients admitted with an acute disease to a general hospital. Clin Nutr 2015; 34: 436-442.

3. Newman R, Vilardell N, Clavé P, et al. Effect of bolus viscosity on the safety and efficacy of swallowing and the kinematics of the swallow response in patients with oropharyngeal dysphagia: white paper by the European Society for Swallowing Disorders (ESSD). Dysphagia 2016; 31: 232-249.

4. Wolter NE, Hernandez K, Irace AL, et al. A systematic process for weaning children with aspiration from thickened fluids. JAMA Otolaryngol Head Neck Surg 2018; 144: 51-56.

5. Choi KH, Ryu JS, Kim MY, et al. Kinematic analysis of dysphagia: significant parameters of aspiration related to bolus viscosity. Dysphagia 2011; 26: 392-398.
6. Leder SB, Judson BL, Sliwinski E, et al. Promoting safe swallowing when puree is swallowed without aspiration but thin liquid is aspirated: nectar is enough. Dysphagia 2013; 28: 58-62.

7. Logemann JA, Gensler G, Robbins J, et al. A randomized study of three interventions for aspiration of thin liquids in patients with dementia or Parkinson's disease. J Speech Lang Hear Res 2008; 51: 173-183.

8. Steele CM, Alsanei WA, Ayanikalath $S$, et al. The influence of food texture and liquid consistency modification on swallowing physiology and function: a systematic review. Dysphagia 2015; 30: 2-26.

9. Lazenby-Paterson T. Thickened liquids: do they still have a place in the dysphagia toolkit? Curr Opin Otolaryngol Head Neck Surg 2020; 28: 145-154.

10. Mandell LA, Niederman MS. Aspiration pneumonia. N EnglJ Med 2019; 380: 651-663.

11. Beck AM, Kjaersgaard A, Hansen T, et al. Systematic review and evidence based recommendations on texture modified

\section{Suggested answers}

1. b.

2. c.

3. b. 
foods and thickened liquids for adults (above 17 years) with oropharyngeal dysphagia - An updated clinical guideline Clin Nutr 2018; 37:1980-1991.

12. Flynn E, Smith CH, Walsh CD, et al. Modifying the consistency of food and fluids for swallowing difficulties in dementia. Cochrane Database Syst Rev 2018; 9: CD011077.

13. Gillman A, Winkler R, Taylor NF. Implementing the free water protocol does not result in aspiration pneumonia in carefully selected patients with dysphagia: a systematic review. Dysphagia 2017; 32: 345-361.

14. Kaneoka A, Pisegna JM, Saito H, et al. A systematic review and meta-analysis of pneumonia associated with thin liquid vs thickened liquid intake in patients who aspirate. Clin Rehabil 2017; 31: 1116-1125.

15. Loeb MB, Becker M, Eady A, et al. Interventions to prevent aspiration pneumonia in older adults: a systematic review. J Am Geriatr Soc 2003; 51: 1018-1022.

16. Robbins J, Gensler G, Hind J, et al. Comparison of 2 interventions for liquid aspiration on pneumonia incidence: a randomized trial. Ann Intern Med 2008; 148: 509-518.

17. Panther K. The Frazier free water protocol. Perspectives on Swallowing and Swallowing Disorders (Dysphagia) 2005; 14: 4-9.

18. Tribst AA, Sant'Ana Ade S, de Massaguer PR. Review: Microbiological quality and safety of fruit juices-past, present and future perspectives. Crit Rev Microbiol 2009; 35: 310-339.

19. Effros RM, Jacobs ER, Schapira RM, et al. Response of the lungs to aspiration. Am J Med 2000; 108: Suppl. 4a, 15s-19s.

20. Chen PH, GolubJS, Hapner ER, et al. Prevalence of perceived dysphagia and quality-of-life impairment in a geriatric population. Dysphagia 2009; 24: 1-6.

21. Swan K, Speyer R, Heijnen BJ, et al. Living with oropharyngeal dysphagia: effects of bolus modification on health-related quality of life-a systematic review. Qual Life Res 2015; 24: 2447-2456.

22. Colodny N. Dysphagic independent feeders' justifications for noncompliance with recommendations by a speech-language pathologist. Am J Speech Lang Pathol 2005; 14: 61-70.
23. McCurtin A, Healy C, Kelly L, et al. Plugging the patient evidence gap: what patients with swallowing disorders poststroke say about thickened liquids. Int J Lang Commun Disord 2018; 53: 30-39.

24. Lim DJ, Mulkerrin SM, Mulkerrin EC, et al. A randomised trial of the effect of different fluid consistencies used in the management of dysphagia on quality of life: a time trade-off study. Age Ageing 2016; 45: 309-312.

25. Cichero JA. Thickening agents used for dysphagia management: effect on bioavailability of water, medication and feelings of satiety. Nutr J 2013; 12: 54.

26. Altman KW, Yu GP, Schaefer SD. Consequence of dysphagia in the hospitalized patient: impact on prognosis and hospital resources. Arch Otolaryngol Head Neck Surg 2010; 136 : 784-789.

27. Murray J, Doeltgen S, Miller M, et al. A survey of thickened fluid prescribing and monitoring practices of Australian health professionals. J Eval Clin Pract 2014; 20: 596-600.

28. Vivanti AP, Campbell KL, Suter MS, et al. Contribution of thickened drinks, food and enteral and parenteral fluids to fluid intake in hospitalised patients with dysphagia. $/$ Hum Nutr Diet 2009; 22: 148-155.

29. Bannerman E, McDermott K. Dietary and fluid intakes of older adults in care homes requiring a texture modified diet: the role of snacks. J Am Med Dir Assoc 2011; 12: 234-239.

30. Finestone HM, Foley NC, Woodbury MG, et al. Quantifying fluid intake in dysphagic stroke patients: a preliminary comparison of oral and nonoral strategies. Arch Phys Med Rehabil 2001; 82: 1744-1746.

31. McCurtin A, Boland P, Kavanagh M, et al. Do stroke clinical practice guideline recommendations for the intervention of thickened liquids for aspiration support evidence based decision making? A systematic review and narrative synthesis. J Eval Clin Pract 2020; 26: 1744-1760.

32. Dobler CC, Harb N, Maguire CA, et al. Treatment burden should be included in clinical practice guidelines. BMJ 2018; 363: k4065. 